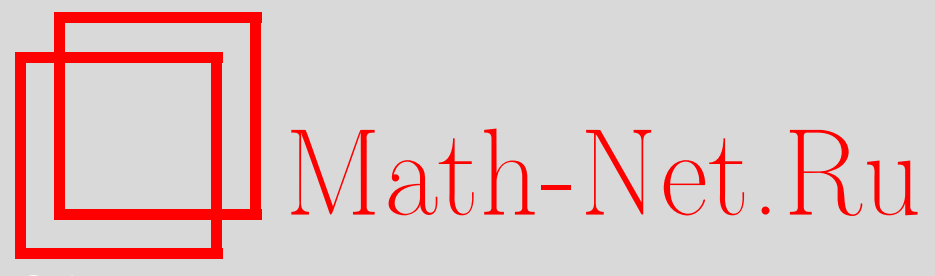

В. А. Кудинов, А. Э. Кузнецова, А. В. Еремин, Е. В. Котова, Аналитические решения квазистатических задач термоупругости с переменными физическими свойствами среды, Вестн. Сам. гос. техн. ун-та. Сер. Физ.-мат. науки, 2014, выпуск 2(), 130-135

DOI: https://doi.org/10.14498/vsgtu1219

Использование Общероссийского математического портала MathNet.Ru подразумевает, что вы прочитали и согласны с пользовательским соглашением

http: //www.mathnet.ru/rus/agreement

Параметры загрузки:

IP : 54.84 .234 .179

26 апреля 2023 г., 14:40:18

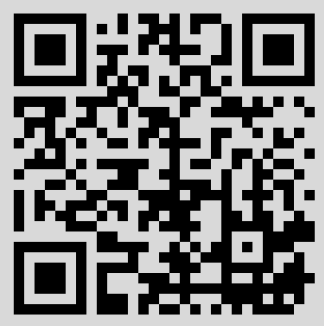


Вестн. Сам. гос. техн. ун-та. Сер. Физ.-мат. науки. 2014. № 2 (35). С. 130-135

УДК 517.958:539.3(4)

\title{
АНАЛИТИЧЕСКИЕ РЕШЕНИЯ КВАЗИСТАТИЧЕСКИХ ЗАДАЧ ТЕРМОУПРУГОСТИ С ПЕРЕМЕННЫМИ ФИЗИЧЕСКИМИ СВОЙСТВАМИ СРЕДЫ
}

\author{
В. А. Кудинов, А. Э. Кузнецова, А. В. Еремин, Е. В. Котова \\ Самарский государственный технический университет, \\ Россия, 443100, Самара, ул. Молодогвардейская, 244.
}

\begin{abstract}
Исполъзуя ортогоналъной метод Бубнова-Галёркина, на основе разработанного метода построения систем координатных функиий, в любом приблиюсении точно удовлетворяющих неоднородным граничным условиям, получено высокой точности приближённое аналитическое решение нелинейной квазистатической задачи термоупругости для бесконечного полого иилиндра с переменными по радиальной координате физическими свойствами. Математическая постановка задачи включает нелинейные уравнения относительно искомой функиии перемещения и неоднородные граничные условия. Решение разыскивается в таком виде, чтобы оно заранее точно удовлетворяло граничным условиям задачи. Точное выполнение граничных условий осуществляется благодаря использованию координатных функиий особой конструкиии. Неизвестные коэффициенты решения находятся путём составления невязки исходного дифференииального уравнения и выполнения требования ортогональности невязки ко всем координатным функииям. Отсюда относительно неизвестных коэффициентов решения получается система алгебраических линейных уравнений, число которых равно числу приближений принятого решения. Показано, что с увеличением числа приближений точность решения существенно возрастает. Так, уже в девятом приближении невязка исходного дифференииального уравнения равна нулю практически во всем диапазоне изменения пространственной переменной. Максимальная невязка в шестом приближении составляет $\varepsilon=5 \cdot 10^{-4}$.
\end{abstract}

Ключевые слова: термоупругость, переменные физические свойства средъ, аналитическое решение, координатные функиии, ортогональный метод БубноваГалёркина.

При скорости изменения температуры, значительно меньшей скорости распространения звука в материале, температурное поле практически не зависит от вызываемых им деформаций. В этом случае можно не учитывать зависящее от деформации слагаемое во взаимосвязанном уравнении тепло-

ISSN: 2310-7081 (online), 1991-8615 (print); doi: http://dx.doi.org/10.14498/vsgtu1219 (C) 2014 Самарский государственный технический университет.

Образец цитирования: В. А. Ку д и н о в, А. Э. Куз не ц о в а, А. В. Е ре ми н, Е. В. Ко то в а, "Аналитические решения квазистатических задач термоупругости с переменными физическими свойствами среды" // Вестн. Сам. гос. техн. ун-та. Сер. Физ.-мат. науки, 2014. № 2 (35). C. 130-135. doi: 10.14498/vsgtu1219.

Сведения об авторах: Василий Александрович Кудинов (д.ф.-м.н., проф.), заведующий кафедрой, каф. теоретических основ теплотехники и гидродинамики. Анастасия Эдуардовна Кузнец,ва, аспирант, каф. теоретических основ теплотехники и гидромеханики. Антон Владимирович Еремин (к.т.н.), старший преподаватель, каф. теоретических основ теплотехники и гидромеханики. Евгения Валериевна Котова (к.т.н.), старший преподаватель, каф. теоретических основ теплотехники и гидромеханики.

E-mail addresses: totig@yandex.ru (V.A. Kudinov), kuznetsovaae@rambler.ru (A.E. Kuznetsova), a.v.eremin@list.ru (A.V. Eremin, Corresponding author), larginaevgenya@mail.ru (E.V. Kotova) 
проводности и инерционные члены в уравнениях равновесия.

Математические постановки задач, в которых не учитываются указанные составляющие термоупругости, называются квазистатическими. В данном случае время теряет смысл переменной и становится параметром, а напряжения определяются исходя из температурного поля в данный конкретный момент времени. В случае учёта указанных факторов задачи термоупругости называются динамическими. Математические постановки таких задач включают сложные неоднородные гиперболические уравнения, методы решения которых пока ещё недостаточно разработаны.

Исходная математическая постановка квазистатической задачи термоупругости включает: уравнения равновесия; уравнения совместности деформаций; соотношения между деформациями и радиальными перемещениями (геометрические уравнения); формулы закона Гука (физические уравнения). Если температура изменяется лишь в радиальном направлении (плоская осесимметричная задача), краевую задачу термоупругости можно свести к задаче о плоском напряжённом состоянии (тонкий круглый диск) или к задаче о плоской деформации (бесконечный цилиндр). При этом исходная математическая постановка задачи, включающая систему дифференциальных уравнений, приводится к одному уравнению относительно искомой функции напряжения или перемещения с соответствующими граничными условиями.

Рассмотрим задачу термоупругости для длинного полого цилиндра в случае его плоской деформации, обусловленной плоским осесимметричным температурным полем. Математическая постановка задачи относительно искомой функции перемещения с переменными по радиальной координате физическими свойствами среды в данном случае имеет вид [1-4]

$$
\begin{gathered}
\frac{d}{d r}\left(E r \frac{d U}{d r}\right)+\left(\frac{\nu}{1-\nu} \frac{d E}{d r}-\frac{E}{r}\right) U=\frac{1+\nu}{1-v} r \frac{d}{d r}(\alpha E T), \\
{\left[(1-\nu) \frac{d U}{d r}+\nu \frac{U}{r}-(1+\nu) \alpha T\right]_{\substack{r=r_{1}, r=r_{2}}}=0,}
\end{gathered}
$$

где $U=U(r)$ - радиальное перемещение; $r$ - радиальная координата; $E=$ $=E(r)$ - модуль упругости; $\alpha=\alpha(r)$ - коэффициент линейного расширения; $\nu$ - коэффициент Пуассона; $T=T(r)$ - температура.

Согласно граничному условию $(2)$, радиальные напряжения $\sigma_{r}(r)$ в точках $r=r_{1}$ и $r=r_{2}$ равны нулю.

При известных перемещениях радиальные и окружные напряжения определяются по формулам

$$
\begin{aligned}
& \sigma_{r}=\frac{E}{(1+\nu)(1-2 \nu)}\left[(1-\nu) \frac{d U}{d r}+\nu \frac{U}{r}-(1+\nu) \alpha T\right], \\
& \sigma_{\theta}=\frac{E}{(1+\nu)(1-2 \nu)}\left[\nu \frac{d U}{d r}+(1-\nu) \frac{U}{r}-(1+\nu) \alpha T\right],
\end{aligned}
$$

где $\sigma_{r}=\sigma_{r}(r)$ - радиальное напряжение; $\sigma_{\theta}=\sigma_{\theta}(r)$ - окружное напряжение.

Трудности получения точного аналитического решения задачи (1), (2) связаны с её нелинейностью. Рассмотрим способ нахождения её приближённого аналитического решения, согласно которому решение задачи (1), (2) при- 
нимается в виде [4]

$$
U(r)=\Phi(r)+\sum_{k=2}^{n} q_{k} \varphi_{k}(r)
$$

где $\Phi(r)=A_{1}+A_{2} r$ - функция, удовлетворяющая неоднородным граничным условиям (2); $A_{1}, A_{2}$ - неизвестные коэффициенты; $\varphi_{k}(r)=1+B_{1} r+B_{2} r^{k}-$ координатные функции, удовлетворяющие однородным граничным условиям (2) (при равенстве нулю слагаемых, содержащих температуру); $B_{1}, B_{2}-$ неизвестные постоянные; $n$ - число приближений.

Для определения постоянных $A_{1}$ и $A_{2}$ используются неоднородные граничные условия (2). Подставляя функцию $\Phi(r)$ в (2), относительно постоянных $A_{1}$ и $A_{2}$ получаем следующую систему двух алгебраических линейных уравнений:

$$
\begin{aligned}
& A_{2}(1-\nu)+\nu \frac{A_{1}+A_{2} r_{1}}{r_{1}}-(1+\nu) \alpha\left(r_{1}\right) T\left(r_{1}\right)=0, \\
& A_{2}(1-\nu)+\nu \frac{A_{1}+A_{2} r_{2}}{r_{2}}-(1+\nu) \alpha\left(r_{2}\right) T\left(r_{2}\right)=0 .
\end{aligned}
$$

Из решения системы уравнений (6) находим

$$
\begin{aligned}
& A_{1}=-\frac{r_{1} r_{2}(1+\nu)\left(\alpha\left(r_{1}\right) T\left(r_{1}\right)-\alpha\left(r_{2}\right) T\left(r_{2}\right)\right)}{\nu\left(r_{1}-r_{2}\right)}, \\
& A_{2}=\frac{(1+\nu)\left(r_{1} \alpha\left(r_{1}\right) T\left(r_{1}\right)-r_{2} \alpha\left(r_{2}\right) T\left(r_{2}\right)\right)}{r_{1}-r_{2}} .
\end{aligned}
$$

Для определения постоянных $B_{1}$ и $B_{2}$ используются граничные условия (2). Положив последний член соотношения (2) равным нулю, относительно неизвестных $B_{1}$ и $B_{2}$ получаем следующую систему двух алгебраических линейных уравнений:

$$
\begin{aligned}
& \left(B_{1}+k B_{2} r_{1}^{k-1}\right)(1-\nu)+\frac{\nu\left(1+B_{1} r_{1}+B_{2} r_{1}^{k}\right)}{r_{1}}=0, \\
& \left(B_{1}+k B_{2} r_{2}^{k-1}\right)(1-\nu)+\frac{\nu\left(1+B_{1} r_{2}+B_{2} r_{2}^{k}\right)}{r_{2}}=0 .
\end{aligned}
$$

Из решения системы уравнений (7) находим

$$
B_{1}=-\frac{\nu\left(r_{2}^{k}-r_{1}^{k}\right)}{r_{1} r_{2}\left(r_{2}^{k-1}-r_{1}^{k-1}\right)}, \quad B_{2}=\frac{\nu\left(r_{2}-r_{1}\right)}{r_{1} r_{2}\left(r_{2}^{k-1}-r_{1}^{k-1}\right)(k-k \nu+\nu)} .
$$

После нахождения неизвестных постоянных $A_{1}, A_{2}, B_{1}, B_{2}$ соотношение (5) будет точно удовлетворять граничным условиям (2). Неизвестные коэффициенты $q_{k}, k=2,3, \ldots, n$, находятся из выполнения уравнения (1). Для этого составляется его невязка и требуется ортогональность невязки ко всем координатным функциям $\varphi_{j}(r), j=2,3, \ldots, n$ :

$$
\int_{r_{1}}^{r_{2}}\left[\frac{d U}{d r}\left(E r \frac{d U}{d r}\right)+\left(\frac{\nu}{1-\nu} \frac{d E}{d r}-\frac{E}{r}\right) U-\frac{1+\nu}{1-\nu} r \frac{d}{d r}(\alpha E T)\right] \varphi_{j}(r) d r=0 .
$$




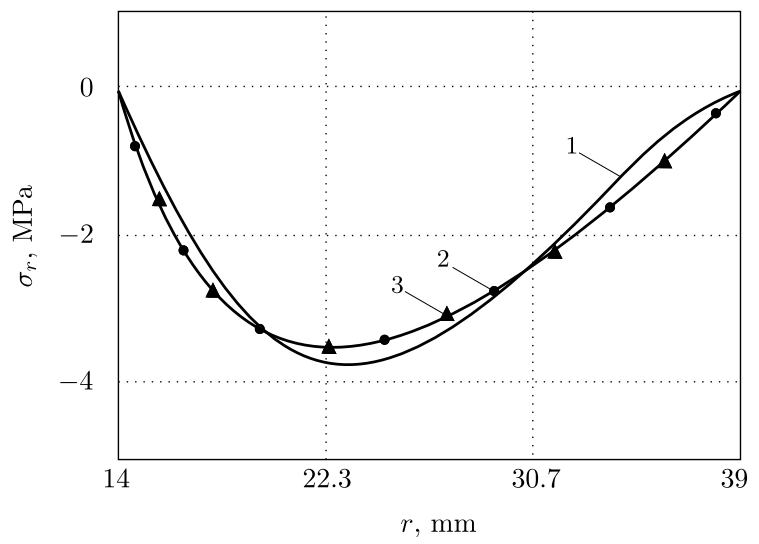

Рис. 1. Эпюры радиальных напряжений (расчёт по формуле (3)): 1 -третье приближение; 2 - шестое приближение; 3 - девятое приближение

[Figure 1. The diagrams of the radial stresses (calculation by formula (3)): 1 - the third approximation; 2 - the sixth approximation; 3 - the ninth approximation]

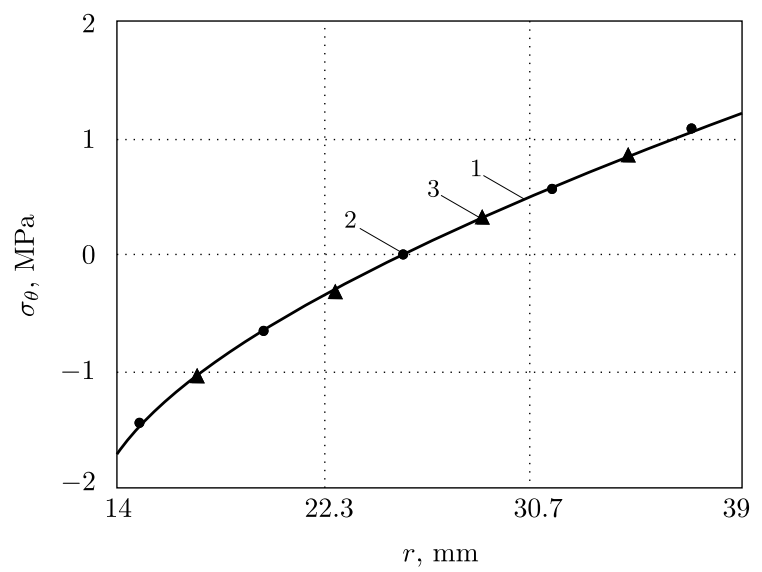

Рис. 2. Эпюры окружных напряжений (расчет по формуле (4)): 1 -третье приближение; 2 - шестое приближение; 3 - девятое приближение

[Figure 2. The diagrams of the circumferential stresses (calculation by formula (4)): 1 - the third approximation; $2-$ the sixth approximation; $3-$ the ninth approximation]

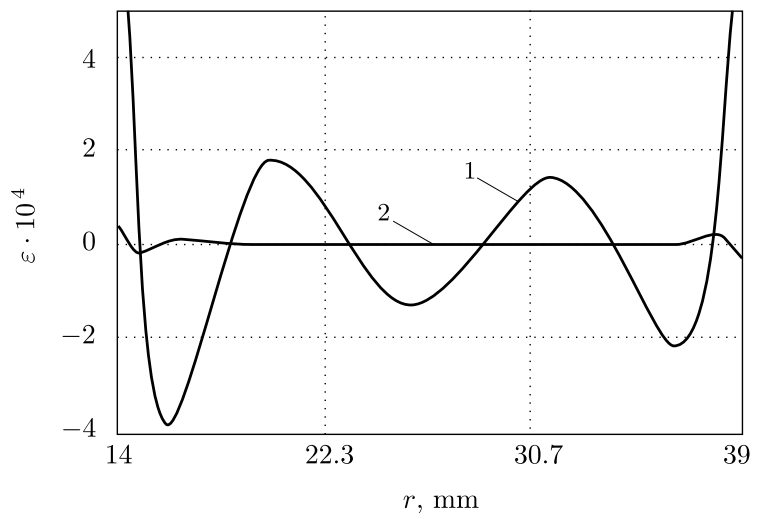

Рис. 3. Невязка уравнения (1): 1 - шестое приближение; 2 - девятое приближение

[Figure 3. The disparity of equation (1): 1 - the sixth approximation; 2 - the ninth approximation] 
Подставляя (5) в (8), после определения интегралов относительно неизвестных коэффициентов $q_{k}$ будем иметь систему $n-2$ алгебраических линейных уравнений. После определения из решения этой системы неизвестных коэффициентов $q_{k}, k=2,3, \ldots, n$, приближённое аналитическое решение задачи (1), (2) находится из (5).

В качестве конкретного примера найдём решение задачи термоупругости для полого цилиндра при следующих исходных данных: $r_{1}=14 \mathrm{mм} ; r_{2}=$ $=39 \mathrm{мм} ; \nu=0.2 ; E=19.5 \cdot 10^{9} \mathrm{Kг} / \mathrm{M}^{2} ; \alpha=10 \cdot 10^{-6} \mathrm{~K}^{-1}$. Распределение температуры по толщине слоя цилиндра принималось в виде

$$
T(r)=170-6592 r+138308 r^{2}-1.7402 \cdot 10^{6} r^{3} .
$$

Результаты расчётов представлены на рис. 1, 2. Их анализ позволяет сделать заключение о том, что как для радиальных, так и для окружных напряжений отмечается незначительное различие результатов шестого и девятого приближений. Следовательно, уже в девятом приближении полученное решение практически совпадает с точным. Оценка невязки уравнения (1) для шестого и девятого приближений подтверждает данное заключение (см. рис. 3). Как видно из рис. 3, невязка уравнения (1) в девятом приближении практически во всем диапазоне изменения переменной $r$ близка к нулю.

Заключение. Разработана методика получения высокоточных приближенных аналитических решений квазистатических нелинейных задач термоупругости (плоская деформация, плоское напряжённое состояние) с переменными физическими свойствами среды. Решение, найденное с помощью ортогонального метода Бубнова-Галёркина при использовании полученной в данной работе системы координатных функций в любом приближении точно удовлетворяющих неоднородным краевым условиям задачи, представляет быстро сходящийся ряд. Так, уже в девятом приближении полученное решение практически совпадает с точным, что подтверждается оценкой невязки основного дифференциального уравнения краевой задачи. Отметим, что ввиду нелинейности этого уравнения точные аналитические решения подобных задач в настоящее время не получены.

Работа выполнена при поддержке Минобрнауки России в рамках базовой части государственного задания ФГБОУ ВПО «СамГТУ». Код проекта: 1273.

This work was supported by the Russian Ministry of Education and Science within the base portion of the state task to Samara State Technical University, project no. 1273.

\section{СПИСОК ЛИТЕРАТУРЫ/ REFERENCES}

1. B. A. Boley, J. H. Weiner, Theory of thermal stresses, New York, John Wiley, 1960, xvi+586 pр.; Б. Боли, Дж. Уэйнер, Теория температурных напряжений. М.: Мир, 1964. 517 c.

2. А. Д. Коваленко, Введение в термоупругость. Киев: Наукова думка, 1965. 202 с. [A. D. Kovalenko, Vvedenie v termouprugost' [Introduction to Thermoelasticity], Kiev, Naukova Dumka, 1965, 202 pp. (In Russian)]

3. S. P. Timoshenko, J. Goodyear, Theory of Elasticity, New York, McGraw-Hill, 1970; С. П. Тимошенко, Дж. Гудьер, Теория упругости. М.: Наука, 1979. 560 с.

4. В. А. Кудинов, Э. М. Карташов, В. В. Калашников, Аналитические решения задач тепломассопереноса и термоупругости для многослойных конструкций. М.: Высшая школа, 2005. 430 с. [V. A. Kudinov, E. M. Kartashov, V. V. Kalashnikov, Analiticheskie resheniya zadach teplomassoperenosa $i$ termouprugosti dlya mnogosloynykh konstruktsiy 
[Analytical problem solving heat and mass transfer and thermoelasticity for multilayer structures], Moscow, Vysshaya shkola, 2005, 430 pp. (In Russian)]

Поступила в редакцию 01/IV/2013;

в окончательном варианте - 05/XII/2013;

принята в печать - 19/III/2014.

MSC: 74F05; 74B20

\title{
ANALYTICAL SOLUTIONS OF THE QUASISTATIC THERMOELASTICITY TASK WITH VARIABLE PHYSICAL PROPERTIES OF A MEDIUM
}

\author{
V. A. Kudinov, A. E. Kuznetsova, A. V. Eremin, E. V. Kotova
}

Samara State Technical University,

244, Molodogvardeyskaya st., Samara, 443100, Russian Federation.

\begin{abstract}
The high-precision approximate analytic solution of the nonlinear quasi-static problem of thermoelasticity for an infinite hollow cylinder with variable along the radial coordinate physical properties is obtained using the orthogonal Bubnov-Galyorkin method developed by the construction of systems of coordinate functions exactly satisfying inhomogeneous boundary conditions in any approximation. The mathematical formulation includes non-linear equations for the unknown function of displacement and inhomogeneous boundary conditions. The desired solution is supposed to precisely satisfy the boundary conditions in advance. The exact fulfillment of the boundary conditions is achieved using the coordinate functions of special design. The unknown coefficients are found by constructing the disparity of original differential equation, that should be orthogonal to all the coordinate functions. Hence, the unknown coefficients of solution yields a system of linear algebraic equations, which number is equal to the number of approximations of the solution. It is shown that the solution accuracy increases substantially with increasing the number of approximations. Thus, already in the ninth approximation the disparity of original differential equation is zero almost the entire range of the spatial variable. The maximum disparity in the sixth approximation is $\varepsilon=5 \cdot 10^{-4}$.
\end{abstract}

Keywords: thermoelasticity problem, variable physical properties of a medium, analytical solution, coordinate functions, Bubnov-Galyorkin orthogonal method.

Received 01/IV/2013;

received in revised form $05 / \mathrm{XII} / 2013$;

accepted 19/III/2014.

ISSN: 2310-7081 (online), 1991-8615 (print); doi: http://dx.doi.org/10.14498/vsgtu1219 (C) 2014 Samara State Technical University.

Citation: V. A. Kudinov, A. E. Kuznetsova, A. V. Eremin, E. V. Kotova, "Analytical Solutions of the Quasistatic Thermoelasticity Task with Variable Physical Properties of a Medium", Vestn. Samar. Gos. Tekhn. Univ., Ser. Fiz.-Mat. Nauki [J. Samara State Tech. Univ., Ser. Phys. \& Math. Sci.], 2014, no. 2 (35), pp. 130-135. doi: 10.14498/vsgtu1219. (In Russian) Authors Details: Vasiliy A. Kudinov (Dr. Phys. \& Math. Sci.), Head of Dept., Dept. of Theoretical Fundamentals of Heat-Engineering and Hydromechanics. Anastasiya E. Kuznetsova, Postgraduate Student, Dept. of Theoretical Fundamentals of Heat-Engineering and Hydromechanics. Anton V. Eremin, Assistant (Cand. Techn. Sci.), Dept. of Theoretical Fundamentals of Heat-Engineering and Hydromechanics. Eugeneya V. Kotova, Assistant (Cand. Techn. Sci.), Dept. of Theoretical Fundamentals of Heat-Engineering and Hydromechanics.

E-mail addresses: totig@yandex.ru (V.A. Kudinov), kuznetsovaae@rambler.ru (A.E. Kuznetsova), a.v.eremin@list.ru (A.V. Eremin, Corresponding author), larginaevgenya@mail.ru (E.V. Kotova) 